\title{
ARTICLE OPEN \\ Spatial distribution of IL4 controls iNKT cell-DC crosstalk in tumors
}

Lu Wang ${ }^{1}$, Zhilan Liu ${ }^{1,2}$, Lili Wang ${ }^{1}$, Qielan $\mathrm{Wu}^{1}$, Xiang $\mathrm{Li}^{1}$, Di Xie ${ }^{1}$, Huimin Zhang ${ }^{1}$, Yongdeng Zhang ${ }^{2}$, Lusheng Gu ${ }^{2}$, Yanhong Xue ${ }^{2}$, Ting Yue ${ }^{3}$, Gang Liu ${ }^{4}$, Wei $\mathrm{Ji}^{2}$, Haiming Wei ${ }^{1}$, Tao $\mathrm{Xu}^{2}$ and Li Bai ${ }^{1}$

The spatiotemporal distribution of cytokines orchestrates immune responses in vivo, yet the underlying mechanisms remain to be explored. We showed here that the spatial distribution of interleukin-4 (IL4) in invariant natural killer T (iNKT) cells regulated crosstalk between iNKT cells and dendritic cells (DCs) and controlled iNKT cell-mediated T-helper type 1 (Th1) responses. The persistent polarization of IL4 induced by strong lipid antigens, that is, a-galactosylceramide (aGC), caused IL4 accumulation at the immunological synapse (IS), which promoted the activation of the IL4R-STAT6 (signal transducer and activator of transcription 6) pathway and production of IL12 in DCs, which enhanced interferon- $\gamma$ (IFN $\gamma$ ) production in iNKT cells. Conversely, the nonpolarized secretion of IL4 induced by Th2 lipid antigens with a short or unsaturated chain was incapable of enhancing this iNKT cell-DC crosstalk and thus shifted the immune response to a Th2-type response. The nonpolarized secretion of IL4 in response to Th2 lipid antigens was caused by the degradation of Cdc42 in iNKT cells. Moreover, reduced Cdc42 expression was observed in tumorinfiltrating iNKT cells, which impaired IL4 polarization and disturbed iNKT cell-DC crosstalk in tumors.

Keywords: Spatial distribution; Polarization; IL4; iNKT; DC; Crosstalk; Tumor

Cellular \& Molecular Immunology (2020) 17:496-506; https://doi.org/10.1038/s41423-019-0243-z

\section{INTRODUCTION}

The release of cytokines is highly regulated in both the spatial and temporal dimensions to orchestrate immune responses. Cascades of cytokines promote crosstalk between distinct immune cells and mount appropriate immune responses in the proper location. Different cytokine secretory routes are utilized by immune cells. It has been shown that interferon- $\gamma$ (IFN $\gamma$ ) and interleukin-2 (IL2) in T-helper type 1 (Th1) cells, ${ }^{1}$ IL10 in Th2 cells, ${ }^{1}$ lytic granules in natural killer (NK) cells, ${ }^{2,3}$ IL12 in dendritic cells (DCs), ${ }^{4,5}$ and lysosomes in $\mathrm{B}$ cells ${ }^{6}$ are directed to immunological synapses (ISs). This polarized secretion efficiently delivers their cargo to the IS, which enables specific targeting and promotes crosstalk between two cells forming an IS. ${ }^{1,7}$ On the other hand, multidirectional secretion of tumor necrosis factor-a (TNFa) and IL4 is observed in Th1 and Th2 cells, respectively, which causes the dispersal of cytokines and regulates only the cells able to respond to low concentrations of cytokines. ${ }^{1}$ The factors controlling secretory routes and functional significance of polarized secretion and multidirectional secretion remain unclear. Spatial alteration of cytokine secretory sites would predictably shift immune responses in vivo by modulating the functions of distinct responder cells.

As important immune regulators, invariant NK T (iNKT) cells release both Th1 and Th2 cytokines (IFNY and IL4, respectively) ${ }^{8}$ and bridge the innate and adaptive immune responses upon recognition of lipid antigens presented by CD1d molecules., 90 Altered iNKT cell functions have been reported during the progression of diseases. ${ }^{11,12}$ Under certain conditions, the activation of iNKT cells will cause either Th1- or Th2-biased responses that are critically related to disease progression and intervention. For example, IFNy production by iNKT cells promotes the clearance of pathogens ${ }^{13}$ and inhibits tumor growth. ${ }^{14,15}$ On the other hand, iNKT cells shift their functions toward Th2 responses by producing large amounts of IL4 but small amounts of IFN $\gamma^{16,17}$ and this change favors tumor growth, ${ }^{18}$ but inhibits autoimmune diseases. ${ }^{17,19,20}$ The mechanisms underlying biased iNKT cell functions are not fully understood. Whether cytokine secretory routes regulate iNKT cell-mediated immune responses remains unclear.

Here, we demonstrate that the spatial distribution of IL4 shapes iNKT cell functions. The secretory routes of IL4 in iNKT cells were controlled by the type of lipid antigen. Unlike the most potent lipid antigen a-galactosylceramide (aGC), several lipid antigen variants with a short or unsaturated chain induce Th2-biased immune responses. ${ }^{16}$ aGC maintained the reorientation of the microtubule-organizing center (MTOC) toward the IS and thus stabilized IL4 polarization. Polarized secretion concentrated IL4 at the IS, which promoted the activation of signal transducer and activator of transcription 6 (STAT6) and production of IL12 in DCs

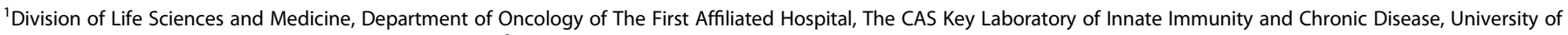

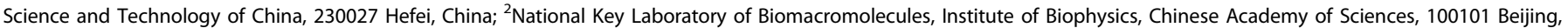

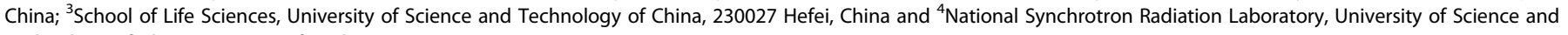
Technology of China, 230027 Hefei, China

Correspondence: Li Bai (baili@ustc.edu.cn)

These authors contributed equally: Lu Wang, Zhilan Liu
}

Received: 16 December 2018 Accepted: 8 May 2019

Published online: 3 June 2019 
and therefore favored IFNy production in iNKT cells. Conversely, multidirectional secretion of IL4 induced by Th2 lipid antigens failed to promote this iNKT cell-DC crosstalk. Taxol stabilized the MTOC and prolonged Th2 lipid antigen-induced IL4 polarization to significantly augment Th1 responses in vitro. Moreover, we showed that multidirectional secretion in response to Th2 lipid antigens resulted from a reduction in $\mathrm{Cdc} 42$ expression. The restoration of active Cdc42 successfully prolonged Th2 lipid antigen-induced polarization of the MTOC and IL4 production. Additionally, the reduction in Cdc42 expression was detected in intratumoral iNKT cells and interfered with IL4 polarization and crosstalk between iNKT cells and DCs in tumors.

\section{RESULTS}

aGC, not Th2 lipid antigen variants, induces activation of the IL4RSTAT6 pathway in DCs

aGC (Fig. 1a) is a strong lipid antigen that activates iNKT cells and induces both IL4 and IFNy production in vivo, whereas $\mathrm{OCH}$, aGC acC8, and aGC acC20:2 (Fig. 1a), which have a truncated or unsaturated acyl chain or sphingosine chain, induce mainly IL4 production in vivo and are therefore regarded as Th2 lipid antigens. $^{21,22}$ Due to reciprocal interactions between iNKT cells and antigen-presenting cells (APCs), the type of APC shapes the functions of the iNKT cells. ${ }^{11,22-24}$ We investigated the interactions between iNKT cells and APCs in vivo. Two hours after injecting aGC, iNKT cells formed clusters exclusively with DCs, especially $\mathrm{CD}^{+}$DCs (Fig. 1b). On the other hand, Th2 lipid antigens are efficiently presented by distinct cell types. ${ }^{22}$ Clusters of iNKT cells around $\mathrm{CD}^{+} \mathrm{DCs}$ were also detected $2 \mathrm{~h}$ after injecting the Th2 lipid antigen aGC acC20:2 (Fig. 1b). Due to the formation of the clusters, it was difficult to count the absolute numbers of iNKT cells. Therefore, we measured the total area occupied by the iNKT cells in a $100 \mu \mathrm{m} \times 100 \mu \mathrm{m}$ square where $\mathrm{CD}^{+} \mathrm{DCs}$ were enriched. The area occupied by the iNKT cells in DC zones was increased $2 \mathrm{~h}$ after injecting aGC. An increased area occupied by iNKT cells in DC zones was also detected after injecting aGC acC20:2 (Fig. 1C). These results indicate that both aGC and the Th2 lipid antigen aGC acC20:2 are able to induce interactions between DCs and iNKT cells. Consistently, similar activation of DCs by aGC and by Th2 lipid antigens, including aGC acC20:2 and $\mathrm{OCH}$, was confirmed, as indicated by comparable upregulation of CD40 and CD86 expression (Fig. 1d). However, aGC and Th2 lipid antigens differed in their capability to induce the activation of STAT6 in DCs in vivo. Only aGC, not Th2 lipid antigens, caused the phosphorylation of STAT6 (Fig. 1e). In agreement with previous findings that indicated that the activation of the STAT6 pathway promotes IL12 production in DCs, ${ }^{25}$ we detected IL12 production in mice injected with aGC, but not in those injected with Th2 lipid antigens (Fig. 1f). The activation of STAT6 is induced by the Th2 cytokines IL4 and IL13. ${ }^{25}$ High expression of IL4 receptor a (IL4Ra) was detected in DCs (Fig. 1g). When splenic DCs were stimulated with lipopolysaccharide (LPS) in vitro, IL4 significantly increased the phosphorylation of STAT6 and production of IL12 in a dosedependent manner (Supplementary Fig. S1A, B), but showed no effect in the expression of costimulatory molecules, including CD40, CD80, and CD86 (Supplementary Fig. S1C). Since iNKT cells produce a large amount of IL4 after activation, it is possible that the activation of STAT6 and production of IL12 in DCs induced by aGC are promoted by IL4 from iNKT cells. Next, we measured the activation of STAT6 in $1 / 4 \mathrm{ra}^{-/-}$mice. IL4R deficiency showed no influence on lipid antigen-induced upregulation of CD40 and CD86 expression in splenic DCs in vivo (Fig. 1h). However, in these II $4 \mathrm{ra}^{-/-}$mice, aGC failed to increase the phosphorylation of STAT6 in the splenic DCs (Fig. 1i). These results confirmed that aGC induced STAT6 activation through IL4R signaling. On the other hand, more IL4 was detected in the serum of these IL4R-deficient mice (Fig. 1j), excluding the possibility that the inhibition of STAT6 activation was due to insufficient IL4 production. In agreement with the role of the IL4-STAT6 pathway in promoting IL12 production, aGC-induced IL12 production was significantly inhibited in the $1 / 4 \mathrm{ra}^{-/-}$mice (Fig. $1 \mathrm{k}$ ). Together, our results demonstrate that lipid antigen variants differ in their capability to activate the IL4R-STAT6 pathway in DCs, which promotes the production of IL12.

IL4 signaling promotes DC-iNKT cell crosstalk and iNKT cellmediated Th1 responses

In agreement with the in vivo studies, when aGC-pulsed DCs were cocultured with iNKT cells, an anti-IL4R antibody and anti-IL4 antibody significantly blocked the activation of STAT6 (Fig. 2a) and reduced the production of IL12 (Fig. 2b) in DCs in vitro. IL12 has previously been shown to promote the Th1 response in iNKT cells. ${ }^{26}$ Here, we found that the reduction in IL12 expression further dampened IFNY production by the iNKT cells in this coculture system, as indicated by reduced IFN $\gamma$ production in the presence of an anti-IL4R-blocking antibody or anti-IL12 antibody (Fig. 2c). To exclude the direct influence of IL4 signaling on iNKT cells, wild-type (WT) bone marrow-derived dendritic cells (BMDCs) and $/ 14 \mathrm{ra}^{-1-}$ BMDCs were generated and transferred into WT recipient mice after the cells were loaded with aGC and carboxyfluorescein succinimidyl ester (CFSE). In comparison to the aGC-loaded WT BMDCs, the aGC-loaded $/ 14 \mathrm{ra}^{-1-}$ BMDCs exhibited less activation of STAT6 (Fig. 2d) and caused less production of IL12 (Fig. 2e) and IFNy (Fig. 2f) in the serum. These results demonstrated that IL4R signaling in DCs was important for DCiNKT cell crosstalk and Th1 responses mediated by iNKT cells.

Distinct secretory sites for IL4 are detected in iNKT cells in response to different lipid antigen variants

Th2 lipids failed to activate the IL4R-STAT6 pathway in DCs (Fig. 1e, i), and this failure was not due to insufficient IL4 production (Fig. 1j). ${ }^{22}$ In addition to the amounts of cytokines, the spatial distributions of cytokines also influence immune responses. To investigate cytokine secretory directions, we labeled the cell surface with a cytokine-specific capture reagent, which captures the cytokine while it is released. Due to the higher local concentration, the cytokine will be more efficiently captured at secretory sites than at other sites (Supplementary Fig. S2A). Thus, a detection antibody specific for the cytokine could be used to indicate the sites of secretion. No signal was detected in cells without cytokine secretion or in the absence of the capture reagent (Supplementary Fig. S2B), which confirmed the specificity of the detection antibody and excluded the possibility of capturing the cytokine by endogenous receptors. The capture reagent labels the cell surface by binding to CD45. ${ }^{27}$ Although CD45 was equally distributed on the cell surface, the cytokine was detected at immune synapses between iNKT cells and APCs (Supplementary Fig. S2B), excluding the possibility that the location of the detection antibody indicated the distribution of CD45 rather than that of the cytokine secretory sites. iNKT cells were cocultured with aGC- or aGC acC8-pulsed DCs, and cytokine secretory sites were detected at the indicated time points with the approach described above. We divided the iNKT cells into four parts with equal interval lines parallel to the synaptic interface, and IL4 release within the first part was defined as polarized secretion (Fig. 3a). Polarized secretion of IL4 at the IS was induced by both aGC and aGC acC 8 after activating cells for $2 \mathrm{~h}$. However, aGC but not aGC acC8 maintained the polarization of IL 4 after $4 \mathrm{~h}$ of activation (Fig. 3b, c). The percentage of iNKT cells that released IL4 at the IS decreased from $85 \%$ to $27 \%$ in response to aGC acC8 (Fig. 3d). When artificial APCs, RBL.CD1d cells, were used instead of DCs to activate iNKT cells, similar results were observed (Fig. 3e). PBS57, another strong lipid antigen that induces a large IFNy response in vivo, ${ }^{28}$ maintained the polarization of IL4 as efficiently as aGC. Even after $8 \mathrm{~h}$ of activation, more than $50 \%$ of the 
a

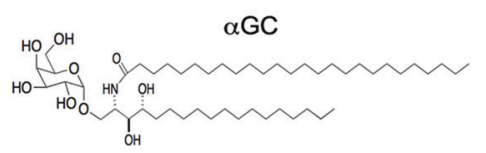

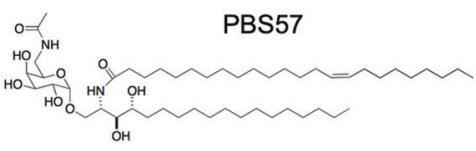

$\alpha G C$ acC20:2

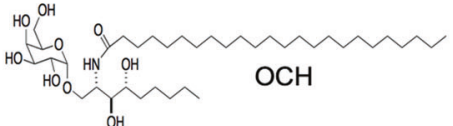

b
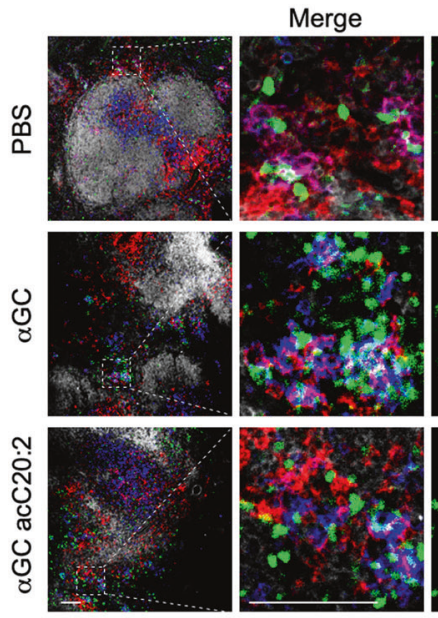

NKT

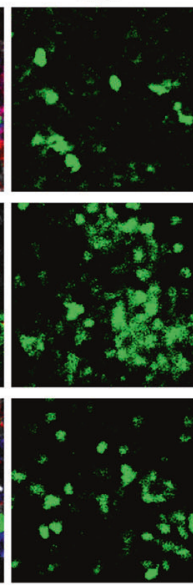

CD8

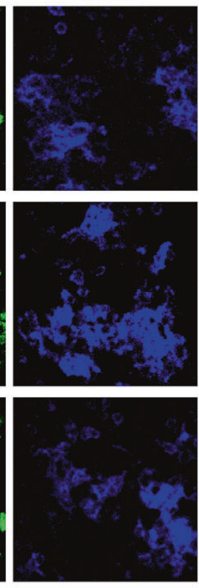

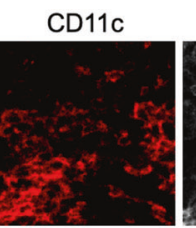
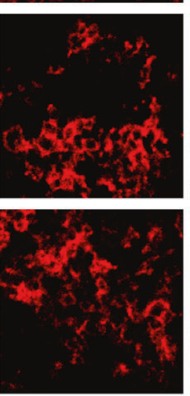
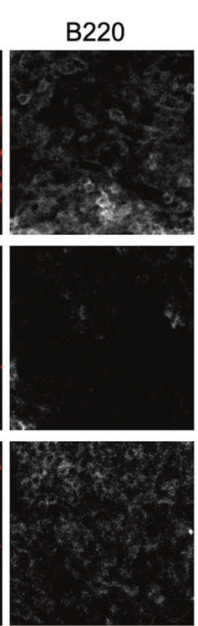

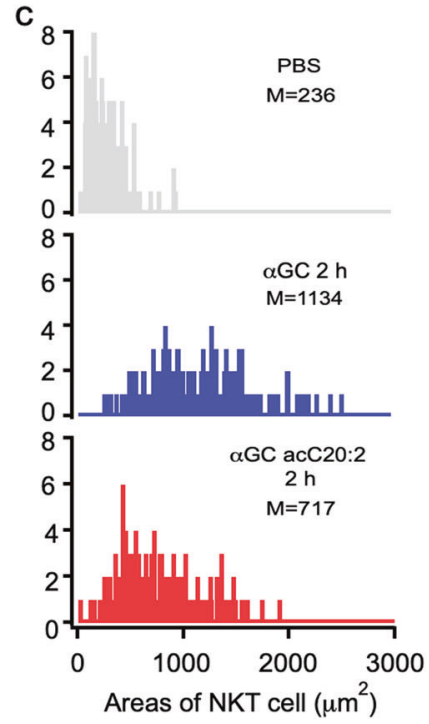

g

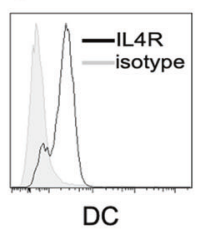

DC
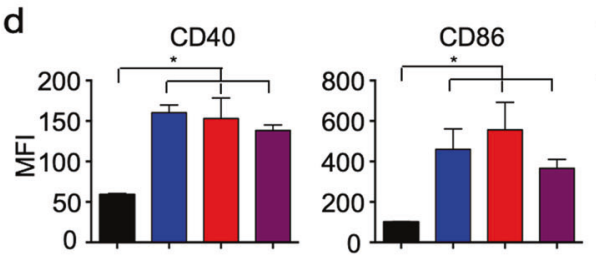

$\mathrm{h}$

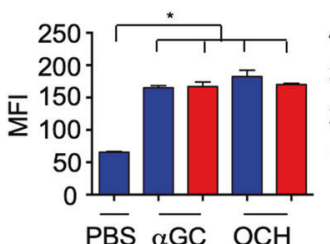

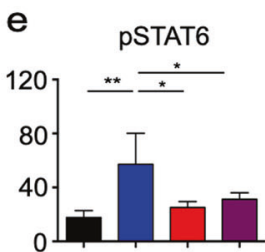

i $\quad$ pSTAT6 f

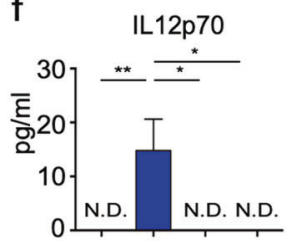

口PS

$\alpha \mathrm{GC}$ acC20:2 $\square \mathrm{OCH}$
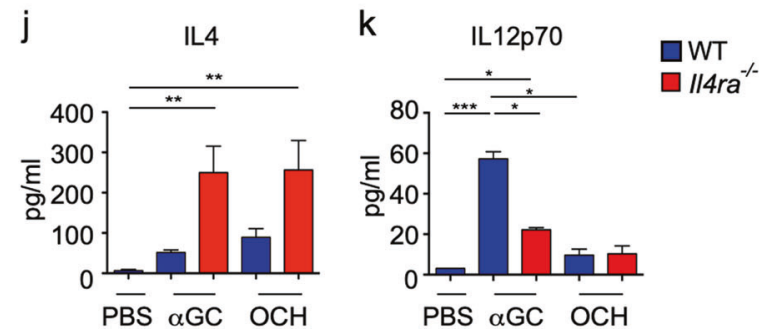

Fig. $1 \alpha$-Galactosylceramide $(\alpha G C)$, not T-helper type 2 (Th2) lipid antigens, activates the IL4 receptor-signal transducer and activator of transcription 6 (IL4R-STAT6) pathway in dendritic cells (DCs.) a Structures of lipid antigen variants. $\mathbf{b}$ In vivo colocalization of invariant natural killer T (iNKT) cells and DCs in the spleen of Va14 Tg. Cxcr6 $6^{f p /+}$ mice $2 \mathrm{~h}$ after injecting $\alpha \mathrm{GC}$ or $\alpha \mathrm{GC}$ acC20:2 (2 $\mu \mathrm{g}$ per mouse, intraperitoneally (i.p.)). Blue, CD8; red, CD11c; gray, B220; and green, iNKT. Scale bars, $50 \mu \mathrm{m}$. Data are representative of three independent experiments. c Distribution of the total area occupied by iNKT cells in each $100 \mu \mathrm{m} \times 100 \mu \mathrm{m}$ DC zone $(n \geq 160$ zones per group). Data are pooled from three independent experiments. M indicates the mean area value. d-f Expression of CD40 and CD86 (d) and phosphorylation of STAT6 (e) in splenic CD11 $\mathrm{c}^{+}$DCs and production of IL12p70 in the serum (f) of wild-type (WT) mice $8 \mathrm{~h}$ after receiving the indicated lipid antigens. Data are presented as the mean \pm SEM of five to six mice per group. g Expression of IL4R $\alpha$ in splenic CD11 ${ }^{+}$DCs. Data are representative of five independent experiments. h-k Expression of CD40 and CD86 (h) and phosphorylation of STAT6 (i) in splenic CD11c ${ }^{+}$DCs and serum production of IL4 (j) and IL12p70 (k) in WT or II4a $a^{-1-}$ mice $8 \mathrm{~h}$ after receiving the indicated lipid antigens. Data are presented as the mean \pm SEM of five to six mice per group. Statistical analysis was performed using one-way analysis of variance (ANOVA) with the Tukey's post test. ${ }^{*} P<0.05 ;{ }^{*} P<0.01 ;$ and ${ }^{* *} P<0.001$

iNKT cells activated by aGC or PBS57 released IL4 in a polarized manner (Fig. 3e). On the other hand, other Th2 lipids, such as aGC acC20:2 and $\mathrm{OCH}$, induced transient polarization of IL4, but multidirectional secretion of IL4 was predominantly detected after activating the iNKT cells for $4 \mathrm{~h}$ (Fig. 3e). To exclude the influence of antigen quantity on cytokine secretory routes, APCs were loaded with different concentrations of aGC or aGC acC8. Our results demonstrated that the type of lipid variants, rather than 

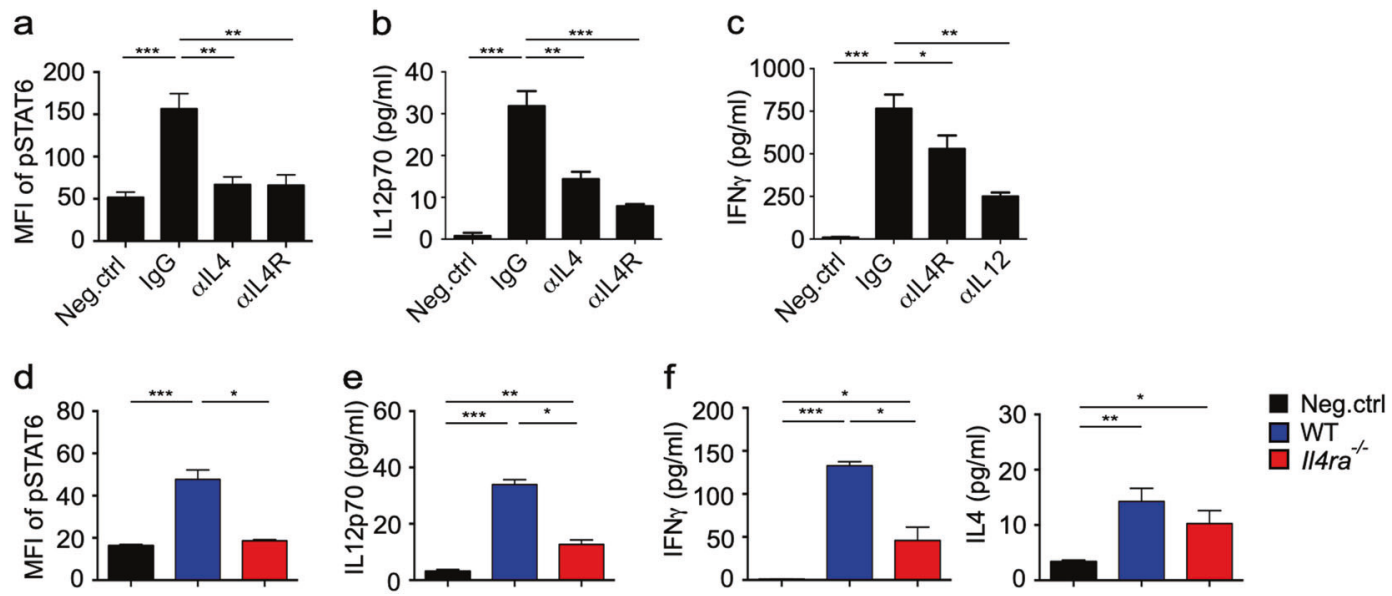

Fig. 2 Interleukin-4 (IL4) promotes invariant natural killer T (iNKT) cell-dendritic cell (DC) crosstalk and T-helper type 1 (Th1) responses. a-c Phosphorylated signal transducer and activator of transcription 6 (STAT6) in CD11 ${ }^{+}$DCs (a) and IL12p70 (b) and IFN $\gamma$ levels (c) in supernatant. iNKT cells were activated for $8 \mathrm{~h}$ by DCs pulsed with $\alpha \mathrm{GC}(1 \mu \mathrm{g} / \mathrm{ml}$ ) with or without immunoglobulin $\mathrm{G}$ (lgG) $2 \mathrm{~b}$ (isotype control), an anti-IL4 antibody, an anti-IL4R antibody, and an anti-IL12 antibody. Data are presented as the mean \pm SEM of more than nine biological replicates. d-f Phosphorylated STAT6 in carboxyfluorescein succinimidyl ester-positive (CFSE ${ }^{+}$BMDCs in the spleen (d) and levels of IL12p70 (e), IFN $\gamma$, and IL4 in the serum (f) of WT recipient mice $8 \mathrm{~h}$ after intravenously (i.v.) injecting $\alpha$-galactosylceramide ( $\alpha \mathrm{GC}$ )-pulsed WT or II4ra ${ }^{-/-}$ $\mathrm{CFSE}^{+}$BMDCs. Data are presented as the mean \pm SEM of five mice per group. Statistical analysis was performed using one-way analysis of variance (ANOVA) with the Tukey's post test. ${ }^{*} P<0.05 ;{ }^{*} P<0.01$; and ${ }^{* *} P<0.001$

a

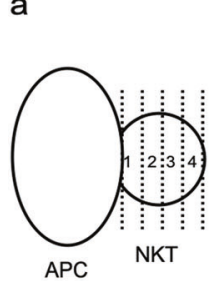

b

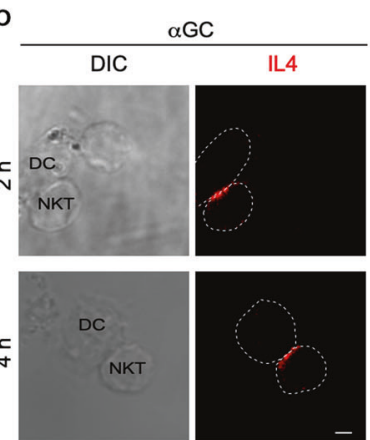

e

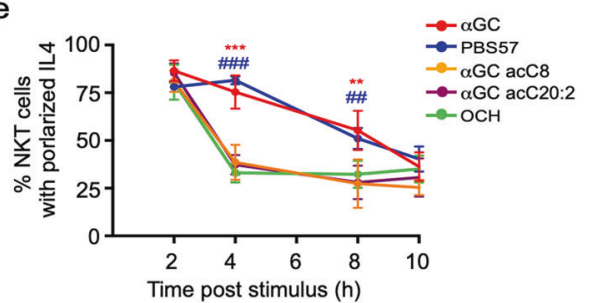

C

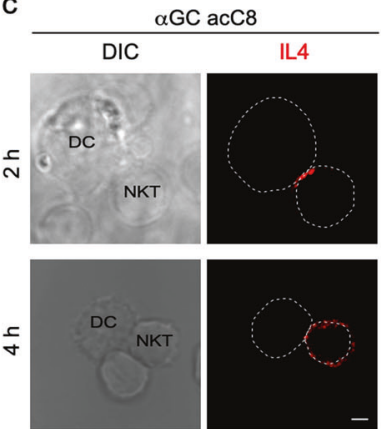

f

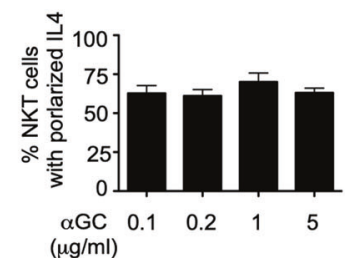

d

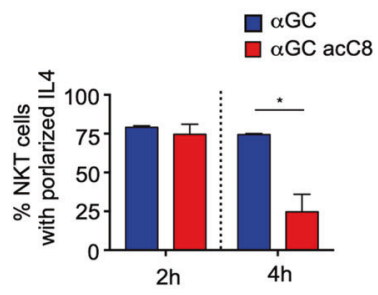

g

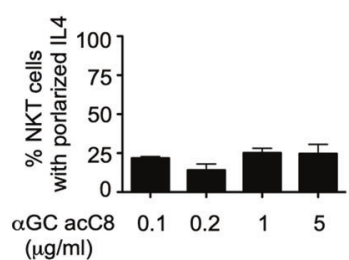

Fig. $3 \alpha$-Galactosylceramide ( $\alpha G C$ ), not T-helper type 2 (Th2) lipid antigens, maintains the polarized secretion of IL4 in invariant natural killer T (iNKT) cells. a Method to quantify the polarized secretion of IL4. IL4 in area 1 is defined as polarized secretion. b, c Secretory sites of IL4 in iNKT cells activated by distinct antigen variant-pulsed splenic dendritic cells (DCs) at the indicated time points. Dotted lines indicate cell boundaries. Scale bars, $2 \mu \mathrm{m}$. Data are representative of three independent experiments and more than 70 cells per group. $\mathbf{d}$ Frequency of iNKT cells with polarized IL4 secretion. Data are presented as the mean \pm SEM of three independent experiments. e Frequency of iNKT cells with polarized IL 4 secretion after activation by distinct antigen variant-pulsed RBL.CD1d cells at the indicated time points. Data are presented as the mean \pm SEM of three independent experiments. * indicates a significant difference between $\alpha G C$ and Th2 lipids, and ${ }^{*}$ indicates a significant difference between PBS57 and Th2 lipids. f, g Frequency of polarized IL4 secretion at the IS induced by the indicated concentrations of $\alpha G C(\mathbf{f})$ and $\alpha G C$ acC $8(\mathbf{g}) 4 \mathrm{~h}$ after activation. Data are presented as the mean \pm SEM of three independent experiments. Statistical analysis was performed using Student's $t$-test, two-way analysis of variance (ANOVA) or one-way ANOVA with the Tukey's post test. ${ }^{*} P<0.05 ;{ }^{* *}$ or ${ }^{\# \#} P<0.01$; and ${ }^{* * *}$ or ${ }^{\# \#} P<0.001$

the amount of lipid, controlled the secretory routes in iNKT cells (Fig. 3f, g).

Different secretory routes activated in response to lipid antigen variants are controlled by the distribution of the MTOC Reorientation of the MTOC toward an IS has been reported to regulate the polarization of vesicles. ${ }^{29-32}$ Next, we investigated the polarization of the MTOC in iNKT cells in response to distinct lipid antigen variants. Polarization of the MTOC reduces the distance from the MTOC to the synaptic interface. ${ }^{33}$ After activating iNKT cells for $2 \mathrm{~h}$, lipid antigens significantly shortened the distance from the MTOC to the synaptic interface, indicating the polarization of the MTOC toward the synaptic interface (Fig. 4a-d). At this time point, the distances from the MTOC to the interface 


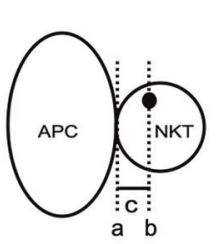

a:SI b:MTOC c:Distance b
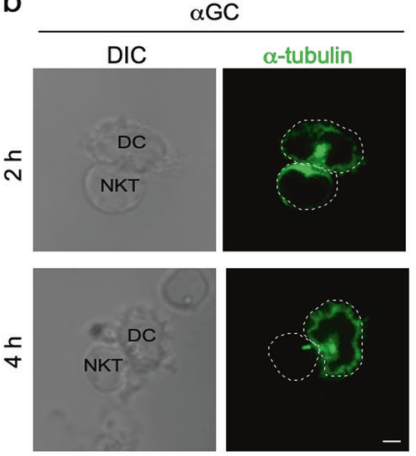

C
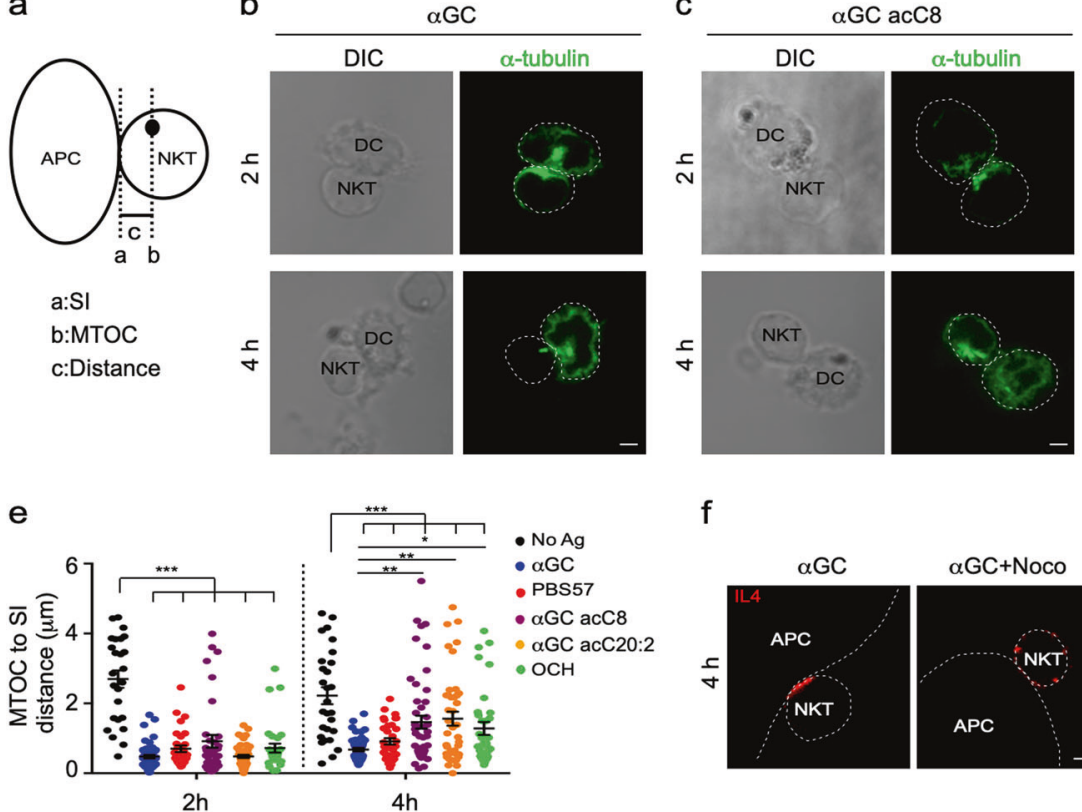

f

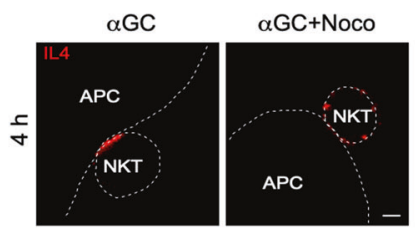

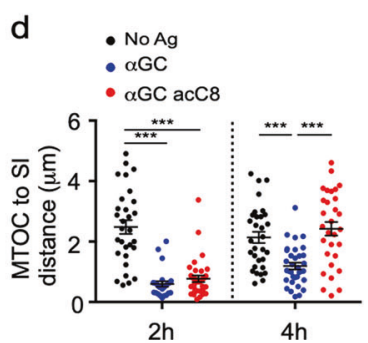

g

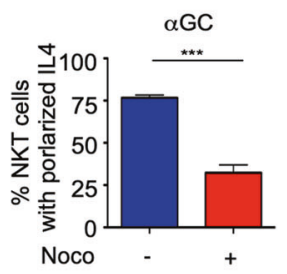

$\mathrm{h}$ $\alpha \mathrm{GC}$ acC20:2 $\quad \alpha \mathrm{GC}$ acC20:2

i + Taxol
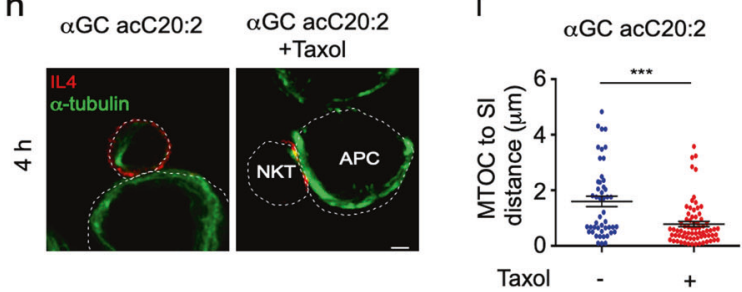

j

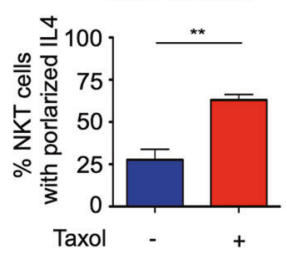

Fig. 4 Microtubule-organizing center (MTOC) controls polarized secretion of interleukin-4 (IL4). a Method to quantify the distance from the MTOC to the synaptic interface (SI). b, $\mathbf{c}$ Polarization of the MTOC in invariant natural killer T (iNKT) cells activated by distinct antigen variantpulsed splenic dendritic cells (DCs) at the indicated time points. Scale bars, $2 \mu \mathrm{m}$. Data are representative of three independent experiments and more than 60 cells per group. $\mathbf{d}$ Distance from the MTOC to the SI in the iNKT cells described in $\mathbf{b}$, $\mathbf{c}$. Data are presented as the mean \pm SEM of more than 40 cells per group. e Distance from the MTOC to the SI in iNKT cells activated by distinct antigen variant-pulsed RBL.CD1d cells at the indicated time points. Data are presented as the mean \pm SEM of more than 60 cells per group. $\mathbf{f}$, $\mathbf{g}$ Influences of nocodazole (33 $\mu M$ ) on IL4 secretory sites (f) and the frequency of iNKT cells with polarized secretion ( $\mathbf{g})$ after activation by $\alpha$-galactosylceramide ( $\alpha G C)$-pulsed RBL. CD1d cells for $4 \mathrm{~h}$. Scale bars, $2 \mu \mathrm{m}$. Data are representative of three independent experiments (f) or are presented as the mean \pm SEM of three independent experiments $(\mathbf{g})$. $\mathbf{h}-\mathbf{j}$ Influences of taxol $(100 \mathrm{nM})$ on the distance from the MTOC to the SI (h, i) and on IL4 polarization (h, $\mathbf{j})$ in iNKT cells activated by $\alpha G C$ acC20:2-pulsed RBL.CD1d cells for $4 \mathrm{~h}$. Scale bars, $2 \mu \mathrm{m}$. Data are representative of three independent experiments (h), are presented as the mean \pm SEM of (j) three independent experiments or are presented as the mean \pm SEM of more than 60 cells per group (i). Dotted lines indicate cell boundaries. Statistical analysis was performed using one-way analysis of variance (ANOVA) with the Tukey's post test or using Student's $t$ test. ${ }^{*} P<0.05 ;{ }^{* *} P<0.01$; and ${ }^{* * *} P<0.001$

were similar between iNKT cells activated by aGC and those activated by the Th2 lipid antigen aGC acC8 (Fig. 4b-d). These results were in agreement with the polarization of IL4 observed at the early time point (Fig. 3b-d). However, after $4 \mathrm{~h}$ of activation, increased distances from the MTOC to the synaptic interface were observed in the iNKT cells activated by aGC acC8 compared to the cells activated by aGC (Fig. 4b-d). The increased distances indicated the redistribution of the MTOC away from the synapse, which was consistent with the multidirectional secretion of IL4 in iNKT cells activated by aGC acC8 (Fig. 3b-d). Moreover, after activating iNKT cells for $4 \mathrm{~h}$, aGC acC20:2 and OCH, but not PBS57, caused a longer distance from the MTOC to the synaptic interface than did aGC (Fig. 4e). These results demonstrated that Th2 lipids were incapable of maintaining the polarization of the MTOC. To investigate whether the MTOC controlled the polarization of IL4, nocodazole, which interferes with the polymerization of microtubules, was added into the culture medium after activating iNKT cells to inhibit aGC-induced MTOC polarization. As a consequence, the percentage of cells with polarized secretion was significantly reduced from 75 to $25 \%$ (Fig. $4 \mathrm{f}$, g). In contrast, when taxol was used to stabilize the MTOC after activating
iNKT cells with aGC acC20:2, the polarization of the MTOC and IL4 was maintained (Fig. $4 \mathrm{~h}-\mathrm{j}$ ). These inhibitors were added $2 \mathrm{~h}$ after $\mathrm{T}$ cell receptor (TCR) engagement to exclude their influence on cell activation. Furthermore, when fixed antigen-pulsed APCs were used to activate iNKT cells, similar results were detected, which excluded an effect of the inhibitors on the APCs (Supplementary Fig. S3). Overall, these results demonstrate that distinct secretory routes in response to lipid variants are controlled by the position of the MTOC in iNKT cells.

Polarization of IL4 at the IS promotes the activation of STAT6 in DCs and iNKT cell-mediated Th1 responses

Although the secretory sites of IL4 in iNKT cells were controlled by lipid antigen variants, the distribution of IL4R on the DC surface was not influenced by these lipids (Fig. 5a). Polarized secretion concentrated IL4 at the IS, as indicated by aGC inducing a higher amount of IL4 at the IS than aGC acC8 (Fig. 5b). Next, we investigated whether the polarization of IL4 promoted crosstalk between DCs and iNKT cells. We forced polarization of IL4 in aGC acC8-activated iNKT cells by adding taxol after cell activation (Fig. 4h, j), and taxol significantly increased the accumulation of 
a
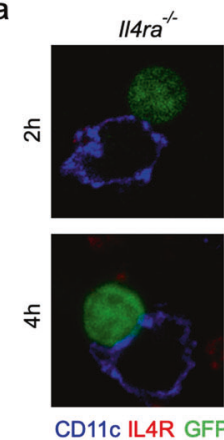

CD11c IL4R GFP (NKT) b

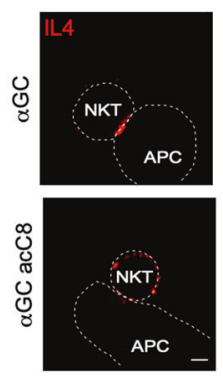

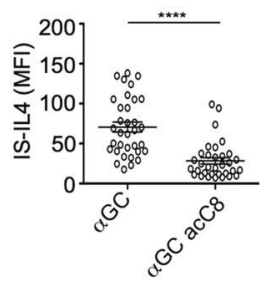

e

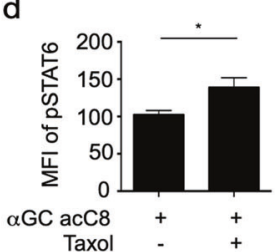

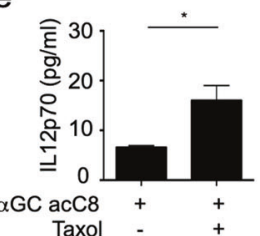

$\mathrm{f}$

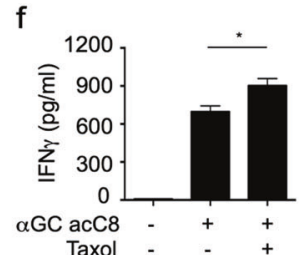

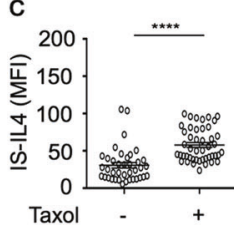

$\mathrm{g}$

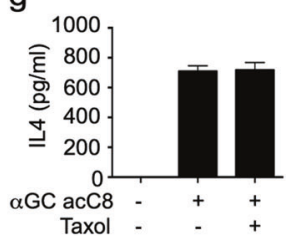

Fig. 5 Taxol prolongs interleukin-4 (IL4) polarization in invariant natural killer T (iNKT) cells and promotes $\alpha$-galactosylceramide ( $\alpha$ GC) acC8induced T-helper type 1 (Th1) responses. a Distribution of IL4R (red) on the surface of CD11 $\mathrm{c}^{+}$(blue) dendritic cells (DCs). iNKT (GFP ${ }^{+}$) cells were activated by distinct antigen variant-pulsed splenic DCs for $4 \mathrm{~h}$. Scale bars, $2 \mu \mathrm{m}$. Data are representative of two independent experiments and more than 25 cells per group. b Accumulation of IL4 at the immunological synapse (IS) in response to $\alpha G C$-pulsed or $\alpha G C$ acC8-pulsed splenic DCs. Dotted lines indicate cell boundaries. Scale bars, $2 \mu \mathrm{m}$. Data are representative of three independent experiments (left) or are presented as the mean \pm SEM of more than 35 cells per group (right). c Influence of taxol (100 nM) on the Th2 lipid-induced accumulation of IL4 at the IS, as shown in Fig. $4 \mathrm{~h}$. $\mathbf{d}-\mathbf{g}$ Influences of taxol (100 nM) on the phosphorylation of signal transducer and activator of transcription 6 (STAT6) in CD11c ${ }^{+}$DCs (d) and on the production of IL12p70 (e), interferon- $\gamma$ (IFN $\gamma$ ) (f), and IL4 (g) in the supernatant. iNKT cells were activated by $\alpha G C$ acC 8 -pulsed $(1 \mu \mathrm{g} / \mathrm{ml})$ DCs for $8 \mathrm{~h}$. Data are presented as the mean \pm SEM of three independent experiments. Statistical analysis was performed using the Mann-Whitney $U$ test. ${ }^{*} P<0.05$ and ${ }^{* * *} P<0.0001$

IL4 at synapses (Fig. 5c). As a result, taxol significantly increased STAT6 phosphorylation (Fig. 5d) and IL12 production (Fig. 5e) in aGC acC8-pulsed DCs. Notably, these results were not due to the direct influence of taxol on the DCs since taxol did not promote the activation of STAT6 and production of IL12 in DCs activated by LPS plus IL4 (Supplementary Fig. S4). Moreover, taxol augmented IFNץ production by iNKT cells (Fig. 5f). The production of IL4 was not changed by taxol (Fig. $5 \mathrm{~g}$ ), which further confirmed that taxol influenced iNKT cell-DC crosstalk by modulating the spatial distribution rather than the amount of IL4. Taken together, our results demonstrate that the polarization of IL4 at the IS favors crosstalk between iNKT cells and DCs and iNKT cell-mediated Th1 responses.

Th2 lipid antigens disturb the polarization of the MTOC and IL4 by reducing the $\mathrm{Cdc} 42$ protein level in iNKT cells

DGK and Cdc42 have been previously shown to regulate MTOC polarization in several cell types. ${ }^{34-36}$ In our studies, an inhibitor of DGK, DGK II, only inhibited the reorientation of the MTOC toward the IS if it was added before iNKT cells were activated by aGCpulsed RBL.CD1d cells (Supplementary Fig. S5A, B). After activation, DGK II showed no influence on the polarization of the MTOC and intracellular IL4 in iNKT cells (Supplementary Fig. S5A, B). Therefore, DGK controlled the initiation of MTOC polarization, but was dispensable for maintaining polarization. On the other hand, ZCL278, an inhibitor of Cdc42, dramatically inhibited the polarization of the MTOC and intracellular IL4 and increased the distance from the MTOC to the synaptic interface, even when added to iNKT cells $3.5 \mathrm{~h}$ after cell activation (Fig. $6 \mathrm{a}, \mathrm{b}$ ). These results indicated a critical role for $\mathrm{Cdc} 42$ in maintaining the polarized distribution of the MTOC at the IS. In agreement with the inability of $\mathrm{OCH}$ to maintain MTOC polarization, a lower amount of GTPbound $\mathrm{Cdc} 42$ was detected in $\mathrm{OCH}$-activated cells than in aGCactivated iNKT cells (Fig. 6c). Importantly, the total Cdc42 protein level was significantly reduced in the OCH-activated iNKT cells but not in the aGC-activated cells (Fig. 6c), which explained the lower activity of $\mathrm{Cdc} 42$ in response to $\mathrm{OCH}$. A similar reduction in the Cdc42 protein level was observed in aGC acC8-activated iNKT cells (Fig. 6d). To test whether the reduction in the Cdc42 level was due to protein degradation, we used GM132 to inhibit proteasome function. MG132 significantly restored the $\mathrm{Cdc} 42$ protein level in OCH-activated iNKT cells (Fig. 6e). Moreover, MG132 successfully prolonged the polarization of the MTOC and IL4 in cells activated by Th2 lipids, including $\mathrm{OCH}$ and aGC acC8 (Fig. $6 \mathrm{f}-\mathrm{k}$ ). To further confirm the influence of the Cdc42 protein level on cytokine secretory routes in iNKT cells, we restored the Cdc42 protein level in cells activated with $\mathrm{OCH}$ by overexpressing $\mathrm{Cdc} 42$. The cells transfected with EGFP-Cdc42 or the constitutively active mutant EGFP-Cdc42V12 but not those transfected with EGFP maintained the polarization of the MTOC and IL4 in response to OCH (Fig. 6I, $\mathrm{m})$. These results proved that recovery of the Cdc42 protein level was able to maintain polarized secretion in response to a Th2 lipid antigen. Notably, the overexpression of the dominant inactive mutant EGFP-Cdc42N17 failed to do so (Fig. 6l, m). Therefore, the activity of Cdc42 was required. Due to the role of Cdc42 in IS formation, ${ }^{35}$ overexpressing EGFP-Cdc42N17 impaired the activation of iNKT cells and thereby reduced the number of IL4expressing iNKT cells (data not shown). Taken together, the results show that lipid antigen variants regulate the secretory routes of IL4 by modulating the protein level of Cdc42.

Reduced Cdc42 expression in intratumoral iNKT cells is associated with impaired IL4 polarization and disturbed iNKT cell-DC crosstalk in tumors

iNKT cell-mediated Th1 responses play important roles in tumor clearance. ${ }^{37}$ Reduced Cdc42 expression was detected in intratumoral iNKT cells compared to splenic iNKT cells from MC38 tumor-bearing mice (Fig. 7a). Consistently, unlike splenic iNKT cells, intratumoral iNKT cells failed to maintain the polarization of IL4 when they were activated by aGC-pulsed RBL.CD1d cells (Fig. 7b, c). Moreover, when 
a

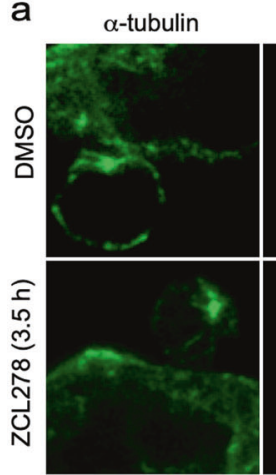

c

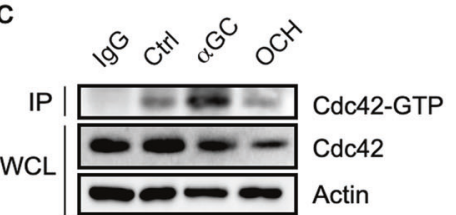

IL4

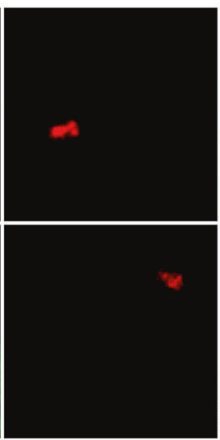

DAPI

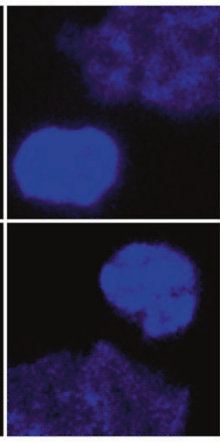

d

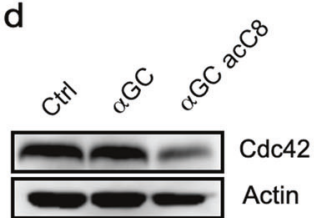

b

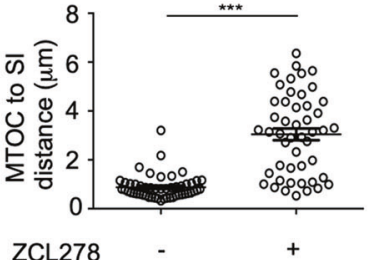

$f$

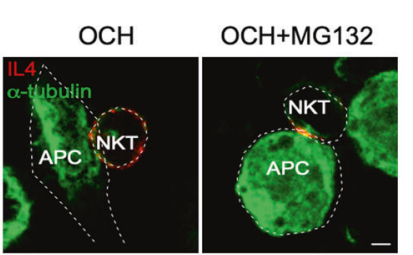

g

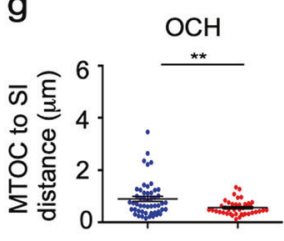

$\mathrm{h}$

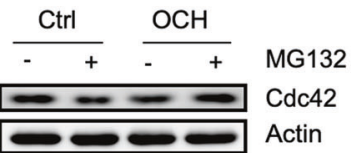

e

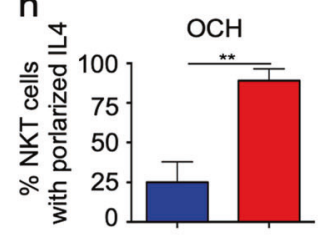

MG132 - +

MG132 - +

k

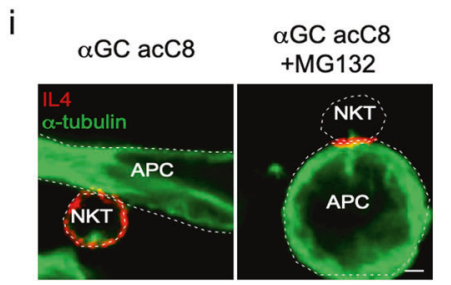

j
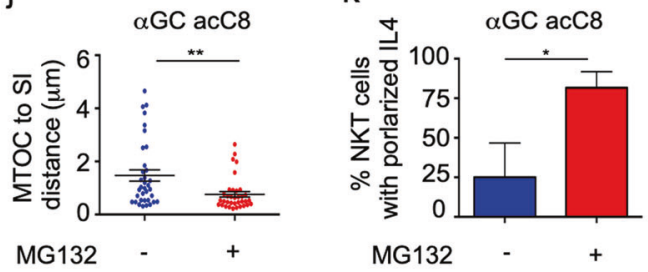

I
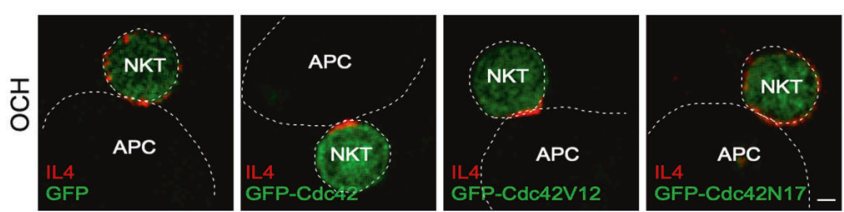

Fig. 6 T-helper type 1 (Th2) lipid antigens disturb the polarization of the microtubule-organizing center (MTOC) by reducing Cdc42 expression in invariant natural killer T (iNKT) cells. a, b Influences of ZCL278 $(100 \mu \mathrm{M})$ on MTOC polarization, intracellular interleukin-4 (IL4) polarization (a), and the distance from the MTOC to the synaptic interface (SI) (b) in iNKT cells activated by $\alpha$-galactosylceramide ( $\alpha G C)$-pulsed RBL.CD1d cells for $4 \mathrm{~h}$. ZCL278 was added to the culture medium in the last $30 \mathrm{~min}$. Scale bars, $2 \mu \mathrm{m}$. Data are representative of three independent experiments (a) or are presented as the mean \pm SEM of more than 45 cells per group (b). $\mathbf{c}$, d Cdc42-GTP and total Cdc42 levels in iNKT cells activated by the indicated lipid antigen-pulsed RBL.CD1d cells for $4 \mathrm{~h}$. Data are representative of three independent experiments. e Influence of MG132 (5 $\mu$ M) on the Cdc42 level in iNKT cells activated by OCH-pulsed RBL.CD1d cells. Data are representative of three independent experiments. $\mathbf{f}-\mathbf{k}$ Influences of MG132 $(5 \mu \mathrm{M})$ on the polarization of the MTOC and IL4 (f, i), the distance from the MTOC to the SI $(\mathbf{g}$, j), and the frequency of iNKT cells with polarized secretion (h, k) after activation by the indicated antigen-pulsed RBL.CD1d cells for $4 \mathrm{~h}$. Scale bars, $2 \mu \mathrm{m}$. Data are representative of three independent experiments (f, i), are presented as the mean \pm SEM of three independent experiments $(\mathbf{h}, \mathbf{k})$, or are presented as the mean \pm SEM of more than 50 cells per group $(\mathbf{g}, \mathbf{j})$. I, $\mathbf{m}$ IL4 secretory sites (I) and the frequencies of iNKT cells overexpressing EGFP-Cdc42, EGFP-Cdc42V12, or EGFP-Cdc42N17 with polarized secretion $(\mathbf{m})$ after activation by OCH-pulsed RBL.CD1d cells for $4 \mathrm{~h}$. Data are representative of three independent experiments (I) or are presented as the mean \pm SEM of $(\mathbf{m})$ three independent experiments. Scale bars, $2 \mu \mathrm{m}$. Dotted lines indicate cell boundaries. Statistical analysis was performed using Student's $t$ test, the Mann-Whitney $U$ test or one-way analysis of variance (ANOVA) with the Tukey's post test. ${ }^{*} P<0.05 ;{ }^{*} P<0.01$; and ${ }^{* * *} P<0.001$ 


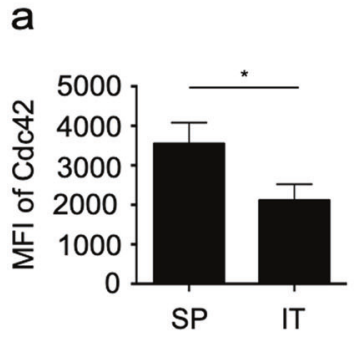

d

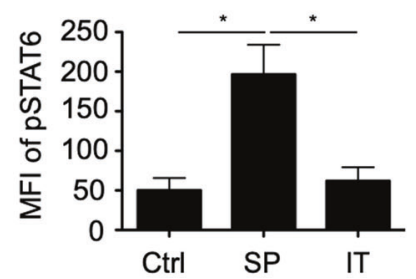

b

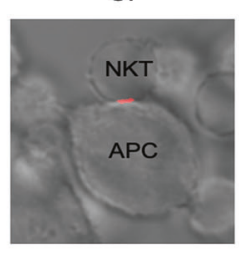

e

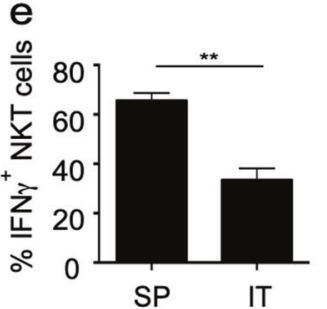

C
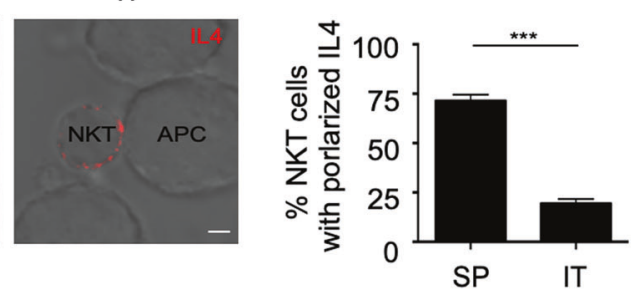

f

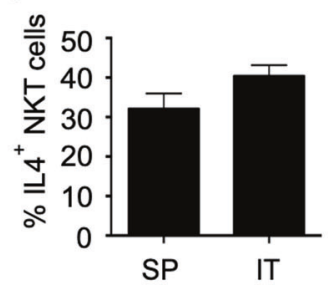

Fig. 7 Impaired interleukin-4 (IL4) polarization and invariant natural killer T (iNKT) cell-dendritic cell (DC) crosstalk in tumors. a Expression of Cdc42 in intratumoral (IT) and splenic (SP) iNKT cells from MC38 tumor-bearing mice. Data are presented as the mean \pm SEM of more than nine mice per group. b, c IL4 secretory sites (b) and the frequency of (c) intratumoral and splenic iNKT cells with polarized secretion after activation by $\alpha$ GC-pulsed RBL.CD1d for $4 \mathrm{~h}$. Scale bars, $2 \mu \mathrm{m}$. Data are representative of three independent experiments (more than 60 cells per group, b) or are presented as the mean \pm SEM of three independent experiments (c). d-f Phosphorylated signal transducer and activator of transcription 6 (STAT6) in splenic DCs and intratumoral MHC II ${ }^{+} \mathrm{CD} 24^{+} \mathrm{F} 4 / 80^{-} \mathrm{CD} 11 \mathrm{c}^{+} \mathrm{DCs}(\mathbf{d})$ and the percentages of IFN $\gamma^{+}(\mathbf{e})$ and IL4 $4^{+}(\mathbf{f})$ iNKT cells in the tumor and spleen of MC38 tumor-bearing mice $8 \mathrm{~h}$ after injection with $\alpha \mathrm{GC}$. Data are presented as the mean \pm SEM of more than nine mice. Statistical analysis was performed using the Mann-Whitney $U$ test or Student's $t$ test. ${ }^{*} P<0.05$; ${ }^{* *} P<0.01$; and ${ }^{* * * P<0.001}$

tumor-bearing mice were injected with aGC, intratumoral DCs and iNKT cells showed less STAT6 phosphorylation (Fig. 7d) and IFNY (Fig. 7e) production, respectively, than cells from the spleen, although similar production of IL4 was detected for the intratumoral iNKT cells and splenic iNKT cells (Fig. 7f). These results were in agreement with the important roles of IL4 polarization in activating STAT6 in DCs and promoting iNKT cell-DC crosstalk. Together, our results suggest that the reduction in $\mathrm{Cdc} 42$ expression in tumorinfiltrating iNKT cells disturbs IL4 polarization and interferes with iNKT cells-DC crosstalk, which contributes to the impaired antitumor Th1 responses mediated by iNKT cells.

\section{DISCUSSION}

IL12 plays an important role in promoting IFN $\gamma$ production in iNKT cells and NK cells. ${ }^{26,38,39}$ Differences in IL12 production explain the biased cytokine profiles in response to lipid antigen variants. ${ }^{40}$ Due to differences in antigen presentation, Th1 lipid antigens are predominantly presented by $\mathrm{CD} 8 \mathrm{a}^{+} \mathrm{DCs}$ that produce a large amount of IL12 and subsequently promote Th1 responses in vivo, whereas Th2 lipid antigens are efficiently presented by multiple CD1d-expressing cell types, most of which are not IL12-producing cells. ${ }^{22}$ However, it is paradoxical that $\mathrm{CD} 8 \mathrm{a}^{+} \mathrm{DCs}$ also present Th2 lipid antigens but fail to produce IL12 (Fig. 1b, f), ${ }^{41}$ and that difference is responsible for the minor IFNy production in response to Th2 lipid antigens. It is well known that iNKT cells promote IL12 production in DCs through CD40LCD40 interactions. ${ }^{42,43}$ However, similar amounts of CD40L and CD40 were expressed by iNKT cells and DCs, respectively, in response to distinct lipid antigen variants (Fig. 1d, Supplementary Fig. S6). Although lipid antigen variants have been shown to modulate the expression of costimulatory and coinhibitory molecules in DCs, these molecules are not involved in regulating the production of IL12. ${ }^{41}$ The mechanisms underlying the differences in IL12 production in response to different lipid antigen variants remain unclear. Our results demonstrate a new way to regulate IL12 production in DCs by modulating cytokine secretory routes. Compared to multidirectional secretion, polarized secretion concentrates cytokines at the cleft between an iNKT cell and APC, which benefits cytokine-mediated cell crosstalk. IL4 promoted IL12 production in DCs in a dosedependent manner (Supplementary Fig. S1B); therefore, the accumulation of IL4 at the iNKT cell-DC IS as a result of polarized secretion favors the activation of the IL4R-STAT6 pathway and the production of IL12 in DCs. In contrast, although Th2 lipid antigen variants were able to induce the production of the same level or even more IL4 than was aGC, the nonpolarized manner of secretion failed to promote IL12 production efficiently and thereby caused Th2-biased cytokine responses. These results demonstrate that cytokines regulate immune responses not only through their amounts but also through their spatial distributions. IL4 is a well-known Th2-promoting cytokine. ${ }^{44,45}$ Paradoxically, IL4 from iNKT cells promoted STAT6 phosphorylation and IL12 production in DCs and enhanced iNKT cell-mediated Th1 responses (Fig. 2). Another study reported that TLR ligandactivated DCs but not helminth Ag SEA-activated DCs increase their IL12 production in response to IL4. ${ }^{25}$ It is possible that the influence of IL4 on DCs is dependent on the activation signaling pathway.

Previous studies indicate different secretory routes for IL4 and IFNy in Th2 and Th1 $\mathrm{CD}^{+}{ }^{+}$T cells, respectively. ${ }^{1}$ It is unclear whether iNKT cells release IL4 and IFN $y$ through different routes. IFNy production exhibited kinetics different from those of IL4 production, and only a small amount of IFN $\gamma$ was detected after activating iNKT cells for $4 \mathrm{~h}$. In the small number of IFNY-positive iNKT cells, the Th1 lipid antigen-induced polarized secretion, whereas the Th2 lipid antigen caused multidirectional secretion (Supplementary Fig. S7A). Interestingly, the release sites of IL4 and IFNY overlapped. Moreover, intracellular IL4 and IFNY were completely colocalized at the IS in iNKT cells activated by aGC (Supplementary Fig. S7B). In agreement with the above results, when nocodazole was used to disturb the polarization of the MTOC, intracellular IL4 vesicles and IFNy vesicles were dispersed around the nucleus and colocalized as well (Supplementary Fig. S7B). Therefore, IL4 and IFN $y$ are released from iNKT cells in the same vesicles. 
Maintaining the polarization of IL4 by taxol treatment significantly enhanced aGC acC8-induced STAT6 phosphorylation (Fig. 5 d) and IL12 production (Fig. 5e) in DCs and IFNy production (Fig. 5f) in iNKT cells. The spatial distributions of cytokines influence cell functions more significantly in vivo than in vitro. Quick diffusion of nonpolarized cytokines is expected in vivo due to extracellular fluid flow, resulting in a low local concentration at the IS. However, in an in vitro system, the accumulation of cytokines in the culture medium would diminish differences in cytokine concentration at the IS caused by distinct secretory routes. These possibilities explain the previous findings that Th2 lipid antigens induce undetectable IL12 production in vivo, but cause a large amount of IL12 production in DCs in vitro after longterm cell culture. ${ }^{22}$ When taxol was used in our studies to stabilize the MTOC and maintain the polarized secretion of IL4 in response to aGC acC8, increased IL12p70 production in DCs was observed only shortly after activation when the concentration of IL4 in the supernatant was low. Moreover, $\mathrm{Cdc} 42$ has been shown to regulate the formation of the IS in several studies. ${ }^{34,35}$ It is possible that lipid antigen variants differ in their ability to maintain IS formation. Again, the IS is less stable in vivo than in vitro due to the high motility of immune cells and fluid shear stress in vivo. It is rational that an unsffig IS induced by Th2 lipid antigens further inhibits the polarization and accumulation of IL4 at the IS, hinders the activation of the IL4R-STAT6 pathway in DCs, and contributes to Th2-biased cytokine responses.

The distinct secretory routes in iNKT cells were controlled by the type of lipid antigen by modulating the protein level of Cdc42 (Fig. 6c, d). Cdc42 but not DGK maintained the reorientation of the MTOC toward the IS in iNKT cells (Supplementary Fig. S5 and Fig. 6a). Th2 lipid antigens induced the degradation of Cdc42 by the proteasome (Fig. 6e). However, the underlying mechanisms are still unclear. Previous studies suggest that lipid antigen variants are distinctly related to lipid raft microdomains in APCs, ${ }^{21,46}$ which might contribute to the different immune responses. ${ }^{46}$ Reduced Cdc42 expression was detected in tumorinfiltrating iNKT cells and was associated with impaired IL4 polarization. In agreement with the observed multidirectional IL4 secretion, diminished STAT6 activation and dampened IFNY production were detected in vivo in intratumoral DCs and iNKT cells, respectively (Fig. 7). These results demonstrated an abnormal secretory route for IL4 and impaired iNKT cell-DC crosstalk in tumors. iNKT cell-mediated Th1 responses play important roles in antitumor immunity. ${ }^{37}$ Therefore, the suppression of iNKT cell-mediated Th1 responses would favor tumor growth. The factors causing the reduction in Cdc42 expression in the tumor microenvironment remain unclear, and whether endogenous lipid antigens are involved and contribute to the progression of tumors requires further investigation.

Overall, our results demonstrate that polarized secretion rather than multidirectional secretion of IL4 in iNKT cells promotes crosstalk between iNKT cells and DCs and enhances iNKT cellmediated Th1 responses (Supplementary Fig. S8). In addition to the amount of a cytokine, the spatial distribution of the cytokine could be another important factor modulating immune responses in vivo.

\section{METHODS}

Mice

C57BL/6 WT mice were purchased from Beijing Vital River Laboratory Animal Technology (Beijing, China). Va14 Tg. Cxcr6 gfp'

+ mice on the C57BL/6 background were provided by Dr. Albert Bendelac (The University of Chicago). ${ }^{22}$ All mice were bred in a specific pathogen-free facility at the University of Science and Technology of China. All animal experiments were approved by the Institutional Animal Care and Use Committee of the University of Science and Technology of China.
Indicated lipid antigens ( $2 \mu \mathrm{g}$ per mouse) were intraperitoneally injected into WT, $/ 14 \mathrm{ra}^{-/-}$, or MC38 tumor-bearing mice. To measure DC activation and cytokine production in the serum, tissue, and serum samples were collected after $8 \mathrm{~h}$. Cytokines produced by intratumoral iNKT cells were measured with cytokine secretion Assay-Detection Kit (Miltenyi Biotec).

Reagents and inhibitors

PBS57, aGC acC8, and aGC acC20:2 were provided by Dr. Albert Bendelac (The University of Chicago) and Dr. Paul B. Savage (Brigham Young University). aGC was purchased from Avanti Polar Lipids, and $\mathrm{OCH}$ was purchased from Cayman Chemical. A CD1dPBS57 tetramer was provided by the NIH Tetramer Core Facility. DGK II, ZCL278, nocodazole, taxol, and MG132 were purchased from Sigma-Aldrich or MedChemExpress.

Cell culture and a cytokine secretion assay

RBL.CD1d cells or splenic DCs were seeded on poly-L-lysine-coated coverslips and pulsed with $1 \mu \mathrm{g} / \mathrm{ml}$ aGC, PBS57, aGC acC8, aGC acC20:2, or $\mathrm{OCH}$ overnight. Then, the cells were cocultured with splenic iNKT cells isolated from Va14 Tg mice with anti-CD4 microbeads (Miltenyi Biotec). To overexpress GFP-Cdc42, GFPCdc42V12, or GFP-Cdc42N17, iNKT cells were transfected using the Amaxa Mouse T Cell Nucleofector ${ }^{\oplus}$ Kit. To study the influences of inhibitors on IL4 secretion, DGK II $(50 \mu \mathrm{M})$ was added a half hour before or $2 \mathrm{~h}$ after activation as indicated. ZCL278 $(100 \mu \mathrm{M})$ and nocodazole $(33 \mu \mathrm{M})$ were added into the culture medium during the last $30 \mathrm{~min}$, taxol $(100 \mathrm{nM})$ was added $2 \mathrm{~h}$ after activation, and MG132 (5 $\mu \mathrm{M})$ was added at the beginning of activation. To detect the secretory sites of IL4 in iNKT cells, the IL4 secretion AssayDetection Kit (Miltenyi Biotec) was used. iNKT cells cocultured with APCs for the indicated time points were labeled with a mouse IL4 capture reagent on ice for $5 \mathrm{~min}$, transferred to a warm medium, and incubated at $37^{\circ} \mathrm{C}$ for $45 \mathrm{~min}$. After washing, the cells were stained with a phycoerythrin (PE)-conjugated anti-mouse IL4 detection antibody for $10 \mathrm{~min}$ on ice.

\section{MTOC polarization assay}

iNKT cells activated by antigen-loaded RBL.CD1d cells or antigenloaded splenic DCs were fixed for $15 \mathrm{~min}$ with $4 \%$ paraformaldehyde. To observe the MTOC, the cells were permeabilized with $0.2 \%$ Triton X-100 (Sigma Aldrich), blocked with 5\% bovine serum albumin (BSA), and then stained with an anti-a-tubulin Alexa Fluor 488-conjugated antibody (Invitrogen) at room temperature for $1 \mathrm{~h}$. For quantification of MTOC polarization, line "a" paralleling the interface between an APC and iNKT cell was drawn at the IS. Line "b" paralleling line "a" was drawn through the MTOC. Then, line " $c$ ", which indicated the vertical distance between line "a" and line "b", was used to quantify MTOC polarity. Images were acquired with an LSM 710 confocal microscope (Zeiss) with a $\times 100$ objective. Data were analyzed with ImageJ software.

\section{Activation of freshly isolated DCs}

DCs were enriched from the spleen of C57BL/6 WT mice with an anti-CD11C PE-conjugated monoclonal antibody and anti-PE microbeads (Miltenyi Biotec). Then, CD11 $\mathrm{c}^{\text {hi }} \mathrm{B} 220^{-}$DCs were sorted by a BD FACS Aria II. GFP hi iNKT cells were sorted from the liver of Va14 Tg. Cxcr6 $6^{g f p /+}$ mice by a BD FACS Aria II. Sorted DCs and iNKT cells were cocultured in 96-well round-bottomed plates in the presence of $1 \mu \mathrm{g} / \mathrm{ml}$ aGC or aGC acC8. LEAF-purified immunoglobulin G (lgG) $2 \mathrm{~b}(5 \mu \mathrm{g} / \mathrm{ml})$, anti-IL4 $(5 \mu \mathrm{g} / \mathrm{ml})$, anti-IL4R $(5 \mu \mathrm{g} / \mathrm{ml})$, and anti-IL12 $(5 \mu \mathrm{g} / \mathrm{ml})$ antibodies were added into the coculture system. After $8 \mathrm{~h}, \mathrm{IL} 12 \mathrm{p} 70, \mathrm{IFN} \gamma$, and IL4 levels in the supernatants were measured by a cytometric bead array (BD Biosciences), and the phosphorylation of STAT6 in DCs was measured by flow cytometry. To investigate the influences of IL4 on DCs, freshly isolated DCs $\left(2 \times 10^{5}\right)$ were stimulated with $1 \mu \mathrm{g} / \mathrm{ml}$ LPS (Sigma-Aldrich) plus murine recombinant IL4 (mrlL4) 
(PeproTech) at the indicated concentrations. All antibodies were purchased from BioLegend or BD Biosciences.

BMDC culture and transfer

BMDCs were generated as previously described. ${ }^{25}$ In brief, total BM cells from WT or $1 / 4 \mathrm{ra}^{-/-}$mice were cultured for 6 days in RPMI medium supplemented with $10 \%$ fetal bovine serum and $\mathrm{mr}$ granulocyte-macrophage colony stimulating factor $(10 \mathrm{ng} / \mathrm{ml}$, PeproTech). Then, the BMDCs were harvested and incubated with or without aGC $(1 \mu \mathrm{g} / \mathrm{ml})$ overnight. After labeling with CFSE (0.2 $\mu \mathrm{M}$, Invitrogen), the BMDCs $\left(1 \times 10^{6}\right.$ cells per mouse) were injected intravenously into WT recipient mice. Tissue and serum samples were collected after $8 \mathrm{~h}$.

\section{Flow cytometry}

Cell surface staining was performed as previously reported. Briefly, cells were blocked with purified anti-CD16/32 and then stained with fluorochrome-conjugated monoclonal antibodies against CD11c (N418), CD11b (M1/70), CD80 (16-10A1), CD86 (24F), CD40 (HM40-3), B220 (RA3-6B2), and TCR $\beta$ (H57-597) and a CD1d-PBS57 tetramer. For pSTAT6 staining, cells were fixed immediately with prewarmed $2 \%$ paraformaldehyde and then permeabilized with ice-cold $90 \%$ methanol. Antibodies against pSTAT6 (CHI2S4N) and CD11c (N418) were incubated with the cells for $1 \mathrm{~h}$ on ice. All antibodies were purchased from eBioscience or BioLegend. The cells were acquired on a FACSVerse flow cytometer (BD Biosciences), and data were analyzed with FlowJo software (TreeStar).

Immunofluorescence microscopy

Va14 Tg. Cxcr6 $6^{g f p /+}$ mice were intraperitoneally immunized with aGC or aGC acC20:2 (2 $\mu \mathrm{g}$ per mouse). Two hours after injection, spleens were harvested, fixed with $4 \%$ paraformaldehyde for $2 \mathrm{~h}$, and then dehydrated in 30\% sucrose overnight before embedding in OCT medium. Frozen sections (20- $\mu$ m thick) were blocked with purified anti-CD16/32 in a 5\% BSA-PBS (phosphate-buffered saline) buffer and then stained with antibodies at $4{ }^{\circ} \mathrm{C}$ overnight. Images were taken with an LSM 710 confocal microscope (Zeiss) with a $\times 10$ objective. Data were analyzed with the ImageJ software.

\section{Western blotting}

RBL.CD1d cells were plated in a six-well plate $\left(1 \times 10^{6}\right.$ cells per well) in the presence of the indicated lipid antigen $(1 \mu \mathrm{g} / \mathrm{ml})$ overnight and then cocultured with hepatic lymphocytes from Va14 Tg. Cxcr6 $6^{g f p /+}$ mice for $4 \mathrm{~h}$. GFP-positive iNKT cells were sorted sequentially and used for western blotting. The antibodies used were as follows: rabbit anti-Cdc42 (Abcam), mouse antiCdc42-GTP (New East), and horseradish peroxidase-conjugated anti-rabbit IgG (Proteintech). To detect GTP-bound Cdc42, iNKT cells were lysed in an NP-40 lysis buffer, and Cdc42-GTP was pulled down by an anti-Cdc42-GTP antibody. Then, GTPbound Cdc42 was detected by an anti-Cdc42 antibody.

\section{Statistical analysis}

Statistical analysis was performed using GraphPad Prism 5. An unpaired Student's $t$ test, the Mann-Whitney $U$ test, one-way analysis of variance (ANOVA) with the Tukey's comparison post test or two-way ANOVA with the Bonferroni correction post test was used to determine significant differences. For all experiments, significance was defined as a $P$ value $<0.05$.

\section{DATA AVAILABILITY}

All data are available within the article (as figure source data or Supplementary Information Files) and/or from the authors on request.

\section{ACKNOWLEDGEMENTS}

We thank the NIH Tetramer Core Facility for providing the CD1d-PBS57 tetramer and Dr. Albert Bendelac and Dr. Paul B. Savage for providing lipid variants. This work was supported by National Key R\&D Program of China 2017YFA0505300, the National Natural Science Foundation of China 91542203 and 81771671, the Strategic Priority Research Program of the Chinese Academy of Sciences XDA12030208, and the Fundamental Research Funds for the Central Universities.

\section{AUTHOR CONTRIBUTIONS}

L.W. and Z.L.L. contributed equally to the paper. L.W., Z.L.L., L.L.W., Q.L.W., X.L., D.X and H.M.Z. performed experiments and data analysis. Y.D.Z., L.S.G., Y.H.X., T.Y., G.L. W.J., H.M.W., and T.X. discussed the experiments and performed data analysis. L.W., Z. L., and L.B. designed the experiments and wrote the manuscript.

\section{ADDITIONAL INFORMATION}

The online version of this article (https://doi.org/10.1038/s41423-019-0243-z) contains supplementary material.

Competing interests: The authors declare no competing interests.

\section{REFERENCES}

1. Huse, M., Lillemeier, B. F., Kuhns, M. S., Chen, D. S. \& Davis, M. M. T cells use two directionally distinct pathways for cytokine secretion. Nat. Immunol. 7, 247-255 (2006).

2. Mace, E. M. et al. NK cell lytic granules are highly motile at the immunological synapse and require F-actin for post-degranulation persistence. J. Immunol. 189, 4870-4880 (2012).

3. Hsu, H. T. et al. NK cells converge lytic granules to promote cytotoxicity and prevent bystander killing. J. Cell Biol. 215, 875-889 (2016).

4. Chiaruttini, G. et al. The SNARE VAMP7 regulates exocytic trafficking of interleukin-12 in dendritic cells. Cell Rep. 14, 2624-2636 (2016).

5. Borg, C. et al. NK cell activation by dendritic cells (DCs) requires the formation of a synapse leading to IL-12 polarization in DCs. Blood 104, 3267-3275 (2004).

6. Yuseff, M. I. et al. Polarized secretion of lysosomes at the B cell synapse couples antigen extraction to processing and presentation. Immunity 35, 361-374 (2011).

7. Tourret, M. et al. T cell polarity at the immunological synapse is required for CD154-dependent IL-12 secretion by dendritic cells. J. Immunol. 185, 6809-6818 (2010).

8. Kronenberg, M. \& Gapin, L. The unconventional lifestyle of NKT cells. Nat. Rev. Immunol. 2, 557-568 (2002)

9. Taniguchi, M., Seino, K. I. \& Nakayama, T. The NKT cell system: bridging innate and acquired immunity. Nat. Immunol. 4, 1164-1165 (2003)

10. Bendelac, A. \& Medzhitov, R. Adjuvants of immunity: harnessing innate immunity to promote adaptive immunity. J. Exp. Med. 195, F19-F23 (2002).

11. Zhang, $H$. et al. M2-specific reduction of CD1d switches NKT cell-mediated immune responses and triggers metaflammation in adipose tissue. Cell Mol. Immunol. 14, 1-12 (2017)

12. Liew, P. X., Lee, W. Y. \& Kubes, P. iNKT cells orchestrate a switch from inflammation to resolution of sterile liver injury. Immunity 47, 752-765 (2017). e755.

13. Brigl, M., Bry, L., Kent, S. C., Gumperz, J. E. \& Brenner, M. B. Mechanism of CD1drestricted natural killer $T$ cell activation during microbial infection. Nat. Immunol. 4, 1230-1237 (2003).

14. Nair, S. \& Dhodapkar, M. V. Natural iller T cells in cancer immunotherapy. Front Immunol. 8, 1178 (2017).

15. Duthie, M. S. et al. During Trypanosoma cruzi infection CD1d-restricted NK T cells limit parasitemia and augment the antibody response to a glycophosphoinositolmodified surface protein. Infect. Immun. 70, 36-48 (2002).

16. $\mathrm{Yu}, \mathrm{K}$. O. et al. Modulation of CD1d-restricted NKT cell responses by using N-acyl variants of alpha-galactosylceramides. Proc. Natl. Acad. Sci. USA 102, 3383-3388 (2005).

17. Miyamoto, K., Miyake, S. \& Yamamura, T. A synthetic glycolipid prevents autoimmune encephalomyelitis by inducing $\mathrm{TH} 2$ bias of natural killer T cells. Nature 413, 531-534 (2001).

18. Tahir, S. M. A. et al. Loss of IFN-gamma production by invariant NK T cells in advanced cancer. J. Immunol. 167, 4046-4050 (2001).

19. Bandyopadhyay, K., Marrero, I. \& Kumar, V. NKT cell subsets as key participants in liver physiology and pathology. Cell Mol. Immunol. 13, 337-346 (2016).

20. Chiba, A. et al. Suppression of collagen-induced arthritis by natural killer T cell activation with $\mathrm{OCH}$, a sphingosine-truncated analog of alphagalactosylceramide. Arthritis Rheum. 50, 305-313 (2004). 
21. Im, J. S. et al. Kinetics and cellular site of glycolipid loading control the outcome of natural killer T cell activation. Immunity 30, 888-898 (2009).

22. Bai, L. et al. Distinct APCs explain the cytokine bias of alpha-galactosylceramide variants in vivo. J. Immunol. 188, 3053-3061 (2012).

23. Bezbradica, J. S. et al. Distinct roles of dendritic cells and B cells in Va14Ja18 natural T cell activation in vivo. J. Immunol. 174, 4696-4705 (2005).

24. Chen, Z. et al. Memory follicular helper invariant NKT cells recognize lipid antigens on memory B cells and elicit antibody recall responses. J. Immunol. 200, 000-000 (2018).

25. Yao, Y. G., Li, W., Kaplan, M. H. \& Chang, C. H. Interleukin (IL)-4 inhibits IL-10 to promote IL-12 production by dendritic cells. J. Exp. Med. 201, 1899-1903 (2005).

26. Tomura, M. et al. A novel function of Va14+CD4+NKT Cells: stimulation of IL-12 production by antigen-presenting cells in the innate immune system. J. Immunol. 163, 93-101 (1999).

27. Guo, L. Y., Li, J. H. \& Paul, W. E. Probabilistic regulation of IL-4 production in Th2 cells: accessibility at the II4 locus. Immunity 20, 193-203 (2004).

28. Liu, Y. et al. A modified a-galactosyl ceramide for staining and stimulating natural killer T cells. J. Immunol. Methods 312, 34-39 (2006).

29. Stinchcombe, J. C. \& Griffiths, G. M. Secretory mechanisms in cell-mediated cytotoxicity. Annu. Rev. Cell Dev. Biol. 23, 495-517 (2007).

30. Stinchcombe, J. C., Majorovits, E., Bossi, G., Fuller, S. \& Griffiths, G. M. Centrosome polarization delivers secretory granules to the immunological synapse. Nature 443, 462-465 (2006)

31. Eng, E. W., Bettio, A., Ibrahim, J. \& Harrison, R. E. MTOC reorientation occurs during FcgammaR-mediated phagocytosis in macrophages. Mol. Biol. Cell 18, 2389-2399 (2007).

32. Martin-Cofreces, N. B. et al. MTOC translocation modulates IS formation and controls sustained T cell signaling. J. Cell Biol. 182, 951-962 (2008).

33. Huse, M. Microtubule-organizing center polarity and the immunological synapse: protein kinase C and beyond. Front. Immunol. 3, 235 (2012).

34. Pulecio, J. et al. Cdc42-mediated MTOC polarization in dendritic cells controls targeted delivery of cytokines at the immune synapse. J. Exp. Med. 207, 2719-2732 (2010).

35. Chemin, K. et al. Cytokine secretion by $\mathrm{CD}^{+} \mathrm{T}$ cells at the immunological synapse requires $\mathrm{Cdc} 42$-dependent local actin remodeling but not microtubule organizing center polarity. J. Immunol. 189, 2159-2168 (2012).

36. Schulz, A. M. et al. Cdc42-dependent actin dynamics controls maturation and secretory activity of dendritic cells. J. Cell Biol. 211, 553-567 (2015).

37. Wolf, B. J., Choi, J. E. \& Exley, M. A. Novel approaches to exploiting invariant NKT cells in cancer immunotherapy. Front Immunol. 9, 384 (2018).
38. Kitamura, H. et al. The natural killer T (NKT) cell ligand alpha-galactosylceramide demonstrates its immunopotentiating effect by inducing interleukin (IL)-12 production by dendritic cells and IL-12 receptor expression on NKT cells. J. Exp. Med. 189, 1121-1128 (1999).

39. Wu, L. S. H. \& Wang, J. Y. Warm up, cool down, and tearing apart in NK cell memory. Cell. Mol. Immunol. 15, 1095-1097 (2018).

40. Oki, S. Preferential Th2 polarization by OCH is supported by incompetent NKT cell induction of $\mathrm{CD} 40 \mathrm{~L}$ and following production of inflammatory cytokines by bystander cells in vivo. Int. Immunol. 17, 1619-1629 (2005).

41. Arora, P. et al. A single subset of dendritic cells controls the cytokine bias of natural killer $\mathrm{T}$ cell responses to diverse glycolipid antigens. Immunity 40, 105-116 (2014).

42. Joyee, A. G., Uzonna, J. \& Yang, X. Invariant NKT cells preferentially modulate the function of CD8 alpha+dendritic cell subset in inducing type 1 immunity against infection. J. Immunol. 184, 2095-2106 (2010).

43. Abdi, K. et al. Cutting Edge: quantitative determination of CD40L threshold for IL-12 and IL-23 production from dendritic cells. J. Immunol. 201, 2879-2884 (2018).

44. Swain, S. L., Weinberg, A. D., English, M. \& Huston, G. IL-4 directs the development of Th2-like helper effectors. J. Immunol. 145, 3796-3806 (1990).

45. Cottagiri, M. et al. In drug-induced, immune-mediated hepatitis, interleukin-33 reduces hepatitis and improves survival independently and as a consequence of FoxP3+T-cell activity. Cell. Mol. Immunol. in press (2018).

46. Bai, L. et al. Lysosomal recycling terminates CD1d-mediated presentation of short and polyunsaturated variants of the NKT cell lipid antigen alphaGalCer. Proc. Natl. Acad. Sci. USA 106, 10254-10259 (2009).

Open Access This article is licensed under a Creative Commons Attribution 4.0 International License, which permits use, sharing, adaptation, distribution and reproduction in any medium or format, as long as you give appropriate credit to the original author(s) and the source, provide a link to the Creative Commons license, and indicate if changes were made. The images or other third party material in this article are included in the article's Creative Commons license, unless indicated otherwise in a credit line to the material. If material is not included in the article's Creative Commons license and your intended use is not permitted by statutory regulation or exceeds the permitted use, you will need to obtain permission directly from the copyright holder. To view a copy of this license, visit http://creativecommons. org/licenses/by/4.0/

(c) The Author(s) 2019 